\title{
Sliding Mode Control of the Vehicle Speed System Based on LMIs
}

\author{
Hong-Liang Gao $\mathbb{D}$, Hong-Cong Zhang $\mathbb{D}$, and Xiao-Ling Li $\mathbb{i}$ \\ School of Electrical Engineering and Automation, Hubei Normal University, Huangshi 435002, China \\ Correspondence should be addressed to Hong-Liang Gao; gaohl2016@hbnu.edu.cn
}

Received 10 February 2021; Revised 17 March 2021; Accepted 12 April 2021; Published 8 June 2021

Academic Editor: Heng Liu

Copyright ( $\odot 2021$ Hong-Liang Gao et al. This is an open access article distributed under the Creative Commons Attribution License, which permits unrestricted use, distribution, and reproduction in any medium, provided the original work is properly cited.

\begin{abstract}
Due to the nonlinear characteristics of the vehicle speed system, its stability is difficult to control. This paper analyzes the stability and traceability of the vehicle speed system under nonlinear characteristics. A sliding mode control method of the nonlinear system state observation based on linear matrix inequalities (LMIs) is proposed. In the proposed control method, Lyapunov function is used as the control function to track the position and speed of the vehicle speed system in real time. In the design process of the controller, the successive scaling method (SSM) is designed to improve the tracking accuracy. The simulation results demonstrate that the sliding mode control can effectively track the position of the vehicle speed system, which has better stability and traceability for the nonlinear vehicle speed system.
\end{abstract}

\section{Introduction}

The stability of the system has always been a key issue in the design of control systems, and its application range is extremely wide. For example, the consistency of multiagent systems and the improvement of power systems are closely related to stability issues [1-5]. In the research of nonlinear systems such as aircrafts, inverted pendulums, and timevarying state-delay systems, some scholars have also conducted in-depth studies on their stability. In [6], the stability of the aircraft is closely related to its performance. In this research, the uncertainty and instability of the aircraft are studied. In [7], the stable tracking for the inverted pendulum system was studied. Due to external interference or certain uncertain factors, it is easy to lose its stability for inverted pendulum systems, so its stability must be ensured. Sliding mode control can be used to control the stability of the system. In [8], a sliding mode control method was proposed to improve the stability of time-varying state-delay systems. However, it is only suitable for small input delay systems. In fact, the study of sliding mode control is based on the dynamic performance of the system itself, such as stability and robustness. Therefore, researchers have begun to pay attention to the research based on sliding mode control and have obtained more and more research results [9-14].
Robust control methods are also widely used in system stability control. In most cases, the robust control problem can be transformed into the problem of solving linear matrix inequalities. For example, linear matrix inequalities are used by some scholars to study underactuated systems $[15,16]$. They designed the observer and improved the robustness of the observer through the coupling method. When the interference is added to the controller, the system cannot maintain the original robustness and stability. In this case, we can also consider using appropriate nonlinear characteristics and reasonable Lyapunov functions to improve the robustness and stability. In short, the application of research based on robust control in real life is significant. In many cases, we can also use other methods to solve the uncertainty of the system. For example, adaptive fuzzy neural network control can be used to solve the uncertainty of the system [17]. In [18], a new adaptive interleaved reinforcement learning algorithm was proposed for nonlinear systems with matching or mismatching uncertainties. It transforms the robust control problem into the optimal control problem of the nominal system and improves the robustness of the system. In [19], coprime decomposition of composite operators was used to solve the robust stability of nonlinear systems with multiple uncertainties. In [20], a multilevel discrete fracture model was presented to guarantee a robust 
and efficient solution for naturally fractured reservoir simulation. In [21], the input-output dynamic stability of networked physical systems was studied. The given networked physical systems are transformed into logical networks with the same robustness. The robustness of networked physical systems is analyzed, and the design method is applied to the robustness analysis of infinite bus systems.

In the control system, the accuracy and robustness of the system are equally important. In order to improve the accuracy of the system, some scholars have studied different control methods to control different systems. A methodology for the synthesis of time-varying static output-feedback gains, capable of stabilising continuous-time linear time-varying systems, was proposed in [22]. In [23], the state feedback nonlinear model predictive control law was approximated offline by the smooth function of the state. This method can improve the accuracy of the system by setting some characteristic parameters. In [24], a new adaptive output feedback control method was proposed to solve the problem of "explosion of complexity" in the case of all state constrained regions based on backstepping. In [25], the control method of the nonlinear system with unknown timevarying disturbance and uncertainty was proposed, which makes the system asymptotically stable in the presence of bounded input and improves the accuracy of the system. In addition, some scholars have designed corresponding control methods to solve the oscillation problem in the system. A method combining empirical mode decomposition with decomposition mode evaluation was proposed in [26]. The method extends the signal preprocessing to improve the robustness and computational efficiency of the system. In [27], an active vibration control technology of the DC motor under harmonic mechanical load torque was introduced. The proposed method can accurately determine the parameters of unknown harmonic vibration. In [28], the problem of linear optimal estimation was studied for discrete and continuous systems with multiple state delays. The linear optimal estimation of the state is obtained by directly calculating the optimal gain, which greatly saves the amount of calculation and increases the accuracy of calculation.

In order to optimize the performance of the system, some researchers have applied different control methods to the converter, the load of the power system, centrifugal compressor, Genesio's chaotic system, and Chua's circuit system. For example, in [29], a simple and systematic approach was presented to design a second-order sliding mode controller for buck converters. The proposed twisting method presents an improvement in steady-state error and settling time of output voltage during load changes. In [30], the load frequency control for power systems with communication delays was studied. The proposed method solves the problem of load disturbance and improves the performance of the system. In [31], a new robust predictive controller was proposed, which used the linear matrix inequality (LMI) tool to improve computation time and complexity. The problem of surge in the centrifugal compressor is solved. In [10], a second-order fast terminal sliding mode control technique based on LMIs was studied. Using this method, a robust chattering-free control scheme was proposed, and the parameters of the controller were given in the form of LMIs. The control structure is independent of the order of the model, and the simulation results in Genesio's chaotic system and Chua's circuit system verify the effectiveness of the scheme. Under these control methods, the corresponding system performance has been improved.

From the above examples, it is not difficult to find that the essence of the research problem is around the robustness and stability of each system. Different controllers can obtain different control effects. Although the aforementioned methods have improved the stability and robustness of the system, most methods do not solve the tracking accuracy problem of the system well, and most strategies are relatively complicated and deserve further investigation.

Inspired by the above discussions, this paper studies the nonlinear characteristics of the vehicle speed system. Roughly, there are three aspects of contributions in this paper:

(i) For the stability control of the vehicle speed system, a sliding mode control method based on the LMI nonlinear state observer is proposed, and the detailed design is given.

(ii) In order to improve the tracking accuracy of the system, the successive scaling method (SSM) is proposed in this paper. In the design of the sliding mode controller, the SSM method is used to make the closed-loop system achieve exponential convergence.

(iii) In the process of solving the gain value of the observer, we obtained the condition of the observer gain value by constructing an inequality and reducing the number of variables.

The rest of the paper is arranged as follows. Some preliminaries and observers' descriptions are presented in Section 2. The main results and derivations of correlation are presented in Section 3. The simulation results and analysis are verified in Section 4. Conclusion is given in Section 5.

\section{Preliminaries}

Consider the vehicle speed system model:

$$
\left\{\begin{array}{l}
\dot{x}_{1}=x_{2}, \\
\dot{x}_{2}=u+h, \\
y=k x_{1},
\end{array}\right.
$$

where $x=\left[\begin{array}{ll}x_{1} & x_{2}\end{array}\right]^{T}, x$ is the state vector, $u$ is the control input, let $u=g \varphi$, and $g$ is a coefficient related to vehicle weight, $\varphi$ represents traction or braking force, $h$ represents the disturbance of the vehicle speed system, $y$ is the system output, and $k$ represents the scale factor of the vehicle position. $x_{1}$ and $x_{2}$ are the position and speed of the vehicle speed system, respectively. At this time, the practical problem can be transformed into the observer-related problem. Let $x_{1}$ follow $x_{t}$, and let $x_{2}$ follow $\dot{x}_{t}$ [32-35].

The state equation of system (1) is

$$
\left\{\begin{array}{l}
\dot{x}=A x+B_{1} u+B_{2} h, \\
y=C x,
\end{array}\right.
$$


where

$$
\begin{aligned}
A & =\left[\begin{array}{ll}
0 & 1 \\
0 & 0
\end{array}\right], \\
B_{1} & =\left[\begin{array}{ll}
0 & 1
\end{array}\right]^{T}, \\
B_{2} & =\left[\begin{array}{ll}
0 & 1
\end{array}\right]^{T}, \\
C & =\left[\begin{array}{ll}
k & 0
\end{array}\right] .
\end{aligned}
$$

$A$ is the system matrix ; $B_{1}$ is the control matrix ; $B_{2}$ is the disturbance correlation matrix; $C$ is the output matrix, where $x=\left[\begin{array}{ll}x_{1} & x_{2}\end{array}\right]^{T}, u$ is the control input, and the designed state observer is

$$
\left\{\begin{array}{l}
\dot{\hat{x}}=A \hat{x}+B_{1} u+B_{2} h+L(y-\hat{y}), \\
\hat{y}=C \hat{x} .
\end{array}\right.
$$

Then,

$$
\left\{\begin{array}{l}
\dot{\hat{x}}_{1}=\widehat{x}_{2}+L_{1}(y-\hat{y}), \\
\dot{\hat{x}}_{2}=u+h+L_{2}(y-\hat{y}), \\
\hat{y}=k \widehat{x}_{1}
\end{array}\right.
$$

where $\hat{x}=\left[\begin{array}{ll}\hat{x}_{1} & \hat{x}_{2}\end{array}\right]^{T}, L=\left[\begin{array}{ll}L_{1} & L_{2}\end{array}\right], L$ denotes the observed gain.

If $\widetilde{x}=x-\widehat{x}$, the following can be obtained:

$$
\begin{aligned}
\dot{\bar{x}} & =\dot{x}-\dot{\bar{x}} \\
& =A x+B_{1} u+B_{2} h-A \hat{x}-B_{1} u-B_{2} h-L(y-\hat{y}) \\
& =(A-L C) \tilde{x} .
\end{aligned}
$$

Define Lyapunov function:

$$
V_{1}=\tilde{x}^{\top} S \tilde{x},
$$

where $S^{\top}=S$, even $S>0$.

The following form can be acquired:

$$
\begin{aligned}
\dot{V}_{1} & =\dot{\tilde{x}}^{T} S \tilde{x}+\tilde{x}^{T} S \dot{\tilde{x}} \\
& =[(A-L C) \tilde{x}]^{T} S \tilde{x}+\tilde{x}^{T} S[(A-L C) \tilde{x}] \\
& =\tilde{x}^{T}(A-L C)^{T} S \tilde{x}+\widetilde{x}^{T} S(A-L C) \tilde{x} \\
& =\tilde{x}^{T}\left[(A-L C)^{T} S+S(A-L C)\right] \tilde{x} .
\end{aligned}
$$

Then let $\mathrm{A}=(A-L C)^{T} S+S(A-L C)$.

Take $\mathrm{A}+\rho I<0, \rho>0$; then

$$
\dot{V}_{1}=\tilde{x}^{T} \mathrm{~A} \tilde{x}<-\rho \tilde{x}^{T} \tilde{x} .
$$

that is,

$$
(A-L C)^{T} S+S(A-L C)+\rho I<0 .
$$

Let $Q=S L$; we have the first LMI as follows:

$$
\left(S A-Q C+[S A-Q C]^{T}\right)+\rho I<0 .
$$

We define $\lambda_{m}$ as the maximum eigenvalue of $\mathrm{A}$.

$$
\begin{aligned}
\dot{V}_{1} & <-\rho \widetilde{x}^{T} \tilde{x} \\
& <-\frac{\rho}{\lambda_{m}} \widetilde{x}^{T} A \widetilde{x}=\gamma \widetilde{x}^{T} A \widetilde{x} \leq \gamma V_{1},
\end{aligned}
$$

where $\gamma=-\rho / \lambda_{m}$.

The solution of inequality $\dot{V}_{1}<-\gamma V_{1}$ is $V_{1}(t)<e^{-\gamma t} V_{1}(0)$, then $V_{1}$ converges to 0 exponentially, and the closed-loop system is exponentially convergent, $t$. The convergence rate of the system depends on $\gamma$.

We know the second LMI is

$$
S>0 .
$$

It follows that

$$
L=S^{-1} Q
$$

\section{Main Results}

In the current section, we select its control law under the premise of sliding mode function and take the control function as Lyapunov function for the vehicle speed system. The successive scaling method is used. Let the observer track the ideal, and we analyze the errors between the actual and the ideal.

Design the sliding mode function:

$$
s=g e+\dot{e} .
$$

For any $g>0, e=x_{t}-x_{1}$, and $\dot{e}=\dot{x}_{t}-\dot{x}_{1}$. Taking the control law of vehicle speed system as

$$
\varphi=\frac{1}{g} \ddot{x}_{t}+\eta \widehat{\mathcal{S}}+\dot{\hat{e}}, \quad(\eta>0),
$$

where $\widehat{s}=g \widehat{e}+\dot{\hat{e}}$, and $\widehat{e}=x_{t}-\widehat{x}_{1}, \dot{\hat{e}}=\dot{x}_{t}-\dot{\hat{x}}_{1}$.

Taking the control Lyapunov function as

$$
V_{2}=\frac{1}{3} s^{3}
$$

due to $\ddot{e}=\ddot{x}_{t}-\ddot{x}_{1}=\ddot{x}_{t}-g \varphi+h$.

$$
\dot{s}=g \dot{e}+\ddot{e}=g \dot{e}+\ddot{x}_{t}-\varphi u-h .
$$

Define $\dot{\tilde{e}}=\dot{e}-\dot{\hat{e}}$ and $\dot{\tilde{x}}_{1}=\dot{x}_{1}-\dot{\hat{x}}_{1}$; the following can be obtained:

$$
\begin{aligned}
\dot{s} & =g \dot{e}+\ddot{x}_{t}-g\left(\frac{1}{g} \ddot{x}_{t}+\eta \widehat{s}+\dot{\vec{e}}\right)-h \\
& =g \dot{\tilde{e}}-g \eta \widehat{s}-h \\
& =g(-\eta s+\eta \widetilde{s})+g \dot{\tilde{e}}-h \\
& =-g \eta s+g \eta\left(-g \widetilde{x}_{1}-\dot{\tilde{x}}_{1}\right)+g(-\dot{\tilde{x}})-h .
\end{aligned}
$$

In this, 


$$
\begin{aligned}
\tilde{x}_{2} & =x_{2}-\widehat{x}_{2}, \\
\widetilde{e} & =e-\widehat{e}=-x_{1}+\widehat{x}_{1}=-\widetilde{x}_{1}, \\
\dot{\tilde{e}} & =\dot{e}-\dot{\hat{e}}=\dot{x}_{t}-\dot{x}_{1}-\left(\dot{x}_{t}-\dot{\tilde{x}}_{1}\right)=-\left(\dot{x}_{1}-\dot{\tilde{x}}\right)=-\widetilde{x}_{1},
\end{aligned}
$$

$$
\widetilde{s}=s-\widehat{s}=g e+\dot{e}-(g \widehat{e}+\dot{\hat{e}})=g \widetilde{e}+\dot{\tilde{e}}=-g \widetilde{x}_{1}-\dot{\tilde{x}}_{1} .
$$

Then, there is

$$
\begin{aligned}
\dot{V}_{2} & =s^{2}\left[-g \eta s+g \eta\left(-g \tilde{x}_{1}-\dot{\tilde{x}}_{1}\right)+g\left(-\dot{\tilde{x}}_{1}\right)-h\right] \\
& =-\left(g \eta s^{3}+s^{2} h\right)+s^{2}\left[-g^{2} \eta \tilde{x}_{1}+(-g \eta-g) \dot{\tilde{x}}_{1}\right] \\
& =-\left(g \eta s^{3}+s^{2} h\right)+k_{1} s^{2} \tilde{x}_{1}+k_{2} s^{2} \dot{\tilde{x}}_{1} .
\end{aligned}
$$

In this, $k_{1}=-g^{2} \eta$ and $k_{2}=-g \eta-g$. According to the properties of the inequality,

$$
\begin{aligned}
& k_{1} s^{2} \tilde{x}_{1} \leq \frac{1}{2} s^{4}+\frac{1}{2} k_{1}^{2} \tilde{x}_{1}^{2}, \\
& k_{2} s^{2} \dot{\tilde{x}}_{1} \leq \frac{1}{2} s^{4}+\frac{1}{2} k_{2}^{2} \dot{\bar{x}}_{1}^{2} .
\end{aligned}
$$

Let $s^{4}<a s^{3}$, the value of $a$ is determined by $s$. Then, we can derive

$$
\begin{aligned}
\dot{V}_{2} & \leq-\left(g \eta s^{3}+s^{2} h\right)+\frac{1}{2} s^{4}+\frac{1}{2} k_{1}^{2} \tilde{x}_{1}^{2}+\frac{1}{2} s^{4}+\frac{1}{2} k_{2}^{2} \dot{\tilde{x}}_{1}^{2} \\
& \leq-\left(g \eta s^{3}\right)+s^{4}+\frac{1}{2} k_{1}^{2} \tilde{x}_{1}^{2}+\frac{1}{2} k_{2}^{2} \dot{\tilde{x}}_{1}^{2} \\
& \leq(-g \eta+a) s^{3}+\eta_{2} \tilde{x}^{\top} \tilde{x} \\
& \leq-\eta_{1} V_{2}+\eta_{2} \tilde{x}^{\top} \tilde{x} .
\end{aligned}
$$

It follows that

$$
\begin{aligned}
\dot{\tilde{x}}_{1}^{2} & =\left(\dot{x}_{1}-\dot{\hat{x}}_{1}\right)^{2} \\
& \leq\left(x_{2}-\hat{x}_{2}-L_{1} C \tilde{x}_{1}\right)^{2} \\
& =\left(\tilde{x}_{2}-L_{1} C \tilde{x}_{1}\right)^{2} \\
& =\tilde{x}_{2}^{2}-2 L_{1} \tilde{x}_{1} C \tilde{x}_{2}+L_{1}^{2} C^{1} \widetilde{x}_{1}^{2} \\
& \leq \tilde{x}_{2}^{2}+L_{1}^{2} C^{2} \tilde{x}_{1}^{2}+L_{1} C \tilde{x}_{1}^{2}+L_{1} C \tilde{x}_{2}^{2} \\
& =\left(1+L_{1} C\right) \tilde{x}_{2}^{2}+\left(L_{1}^{2} C+L_{1}\right) C \tilde{x}_{1}^{2}, \\
\frac{1}{2} k_{1}^{2} \widetilde{x}_{1}^{2}+\frac{1}{2} k_{2}^{2} \dot{\tilde{x}}_{1}^{2} & \leq \frac{1}{2} k_{1}^{2} \tilde{x}_{1}^{2}+\frac{1}{2} k_{2}^{2}\left[\left(1+L_{1} C\right) \tilde{x}_{2}^{2}+\left(L_{1}^{2} C+L_{1}\right) C \widetilde{x}_{1}^{2}\right] \\
& =\left[\frac{1}{2} k_{1}^{2}+\frac{1}{2} k_{2}^{2}\left(L_{1}^{2} C+L_{1}\right) C\right] \tilde{x}_{1}^{2}+\frac{1}{2} k_{2}^{2}\left(1+L_{1} C\right) \tilde{x}_{2}^{2},
\end{aligned}
$$

Where

$\eta_{2}=\max \left\{(1 / 2) k_{1}^{2}+(1 / 2) k_{2}^{2}\left(L_{1}^{2} C+L_{1}\right) C,(1 / 2) k_{2}^{2}\left(1+L_{1} C\right)\right\}$ and $\eta_{1}=3(g \eta-a)$.

The Lyapunov function of the vehicle speed system is

$$
V=V_{1}+V_{2},
$$

where $V_{1}=\tilde{x}^{\top} S \tilde{x}$.

It can be seen from (13) that

$$
\begin{aligned}
\dot{V} & =\dot{V}_{1}+\dot{V}_{2}<-\rho \tilde{x}^{T} \tilde{x}+\eta_{2} \tilde{x}^{T} \tilde{x}-\eta_{1} V_{2} \\
& =-\eta_{1} V_{2}-\left(\rho-\eta_{2}\right) \tilde{x}^{T} \tilde{x} .
\end{aligned}
$$

For $\rho>\eta_{2}$, we can gain

$$
\begin{gathered}
-\left(\rho-\eta_{2}\right) \tilde{x}^{T} \tilde{x}<-\frac{\rho-\eta_{2}}{\lambda_{m}} \tilde{x}^{T} A \tilde{x}=-b \tilde{x}^{T} A \tilde{x}<-b V_{1} . \\
\lambda_{m} \text {, where } b=\left(\rho-\eta_{2}\right) / \lambda_{m}, \rho>\eta_{2} . \text { And Then } \\
\dot{V} \leq-\eta_{1} V_{2}-b V_{1} \leq-\tau\left(V_{1}+V_{2}\right)=-\tau V,
\end{gathered}
$$

where $\tau=\min \left\{\eta_{1}, b\right\}$.

The solution of inequality $\dot{V} \leq-\tau V$ is $V(t) \leq e^{-\tau t}$, then the exponential form of $V(t)$ converges to 0 . When $t \longrightarrow \infty$, $s \longrightarrow 0, \quad \tilde{x} \longrightarrow 0$, the system satisfies the exponential convergence condition, then the vehicle speed system is also exponentially convergent. The convergence rate of the system depends on $\eta_{1}, b$.

The so-called SSM is a method of applying the related principles of inequality scaling to obtain the desired result. Specifically, we first set reasonable equations (15) and (16), and (24) is established according to the related principle of inequality, and then we appropriately scale $V_{2}$ on the basis of (24) to obtain a form related to (17). Finally, combine $V_{1}$ and $V_{2}$ to get (30); it can be concluded that the closed-loop system is exponentially convergent from (30).

\section{Simulation}

Take (2) as the controlled object and (4) as the observer, and the initial state value of the observer is $\hat{x}(0)=\left[\begin{array}{ll}0 & 0\end{array}\right]$. The initial state of the controlled object is $x(0)=\left[\begin{array}{ll}0.5 & 0\end{array}\right]$. By solving LMIs (11) and (13), we obtain the observation gain value $L=\left[\begin{array}{ll}0.9678 & 1.3179\end{array}\right]^{\top}$. In the present study, it is set that $\alpha=29$, and $x 1 p \longrightarrow x 1, x 2 p \longrightarrow x 2$. The simulation results are shown in Figures 1 and 2.

The vehicle speed system is simulated under given conditions. The controlled object is (1), and the initial state is set as $x(0)=\left[\begin{array}{ll}0.5 & 0\end{array}\right]$. The observer is (5), and the initial state of the observer is set as $\hat{x}(0)=\left[\begin{array}{ll}0 & 0\end{array}\right]$. Equation (16) is the controller, and $g=10, \eta=4.0$. The position instruction takes $x_{t}=\cos (t)$. We obtain the observation gain value $L$ by solving inequalities. The simulation results are shown in Figures 3-5.

In Figure 1(a), the tracking effect is poor in the first 2 seconds, and the tracking effect has a higher degree of approximation after 2 seconds. In Figure 1(b), the tracking effect is gradually improved after 5 seconds.

In Figure 2(a), the error gets smaller in the first 10 seconds. The error goes almost to 0 after 10 seconds. In 


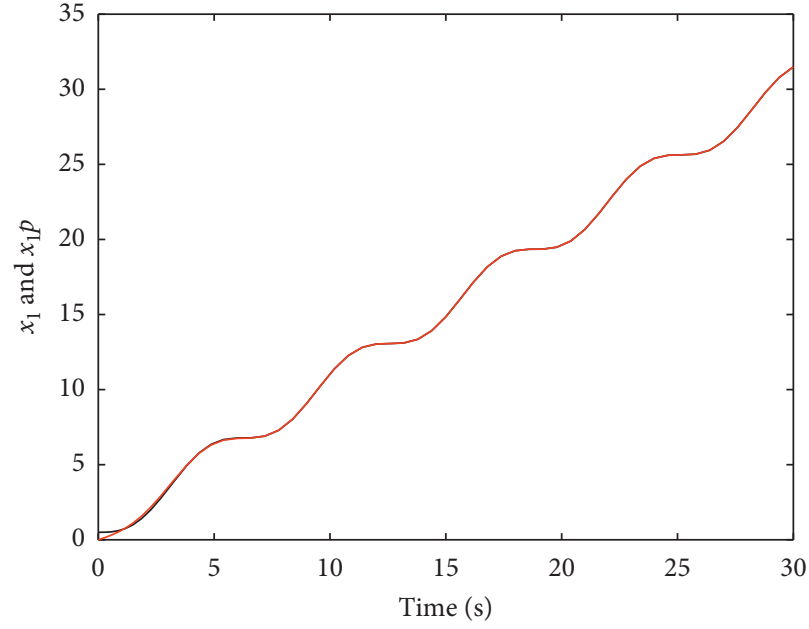

(a)

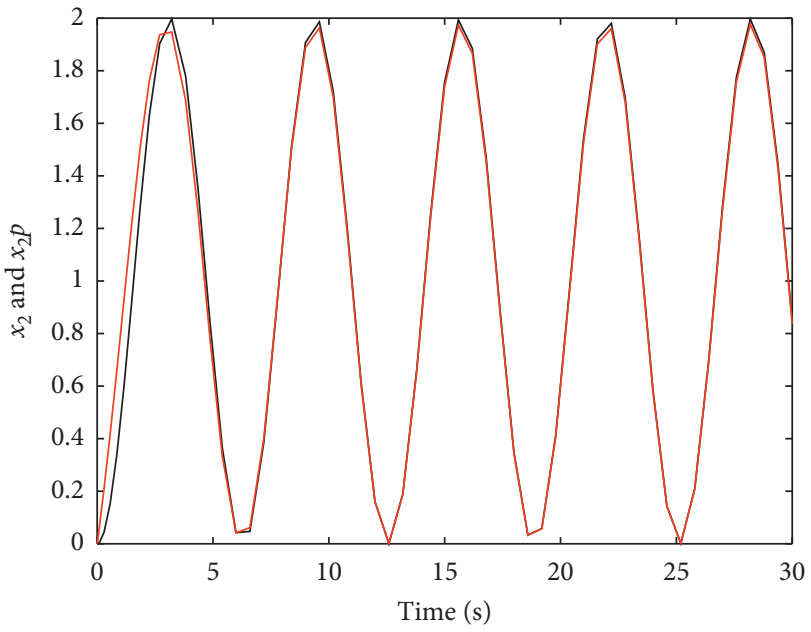

(b)

Figure 1: State observation results.

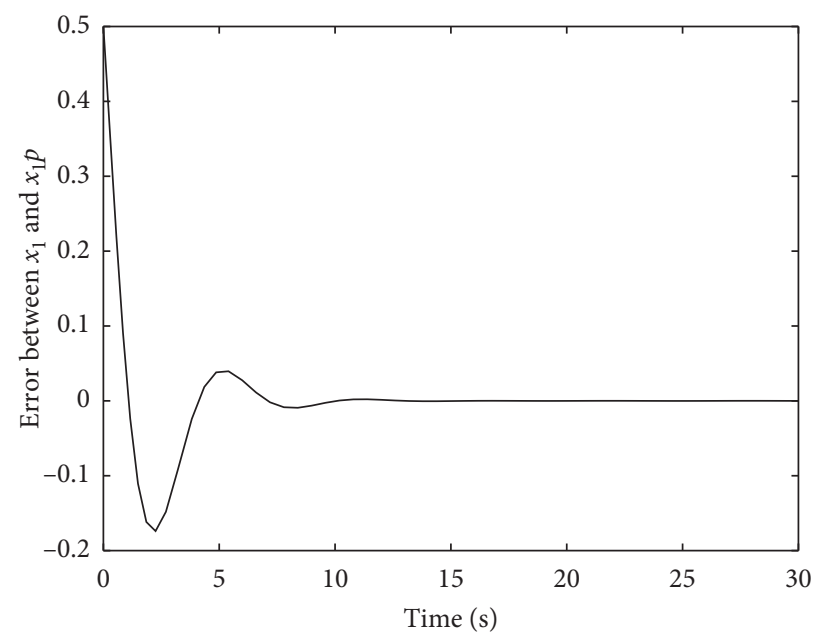

(a)

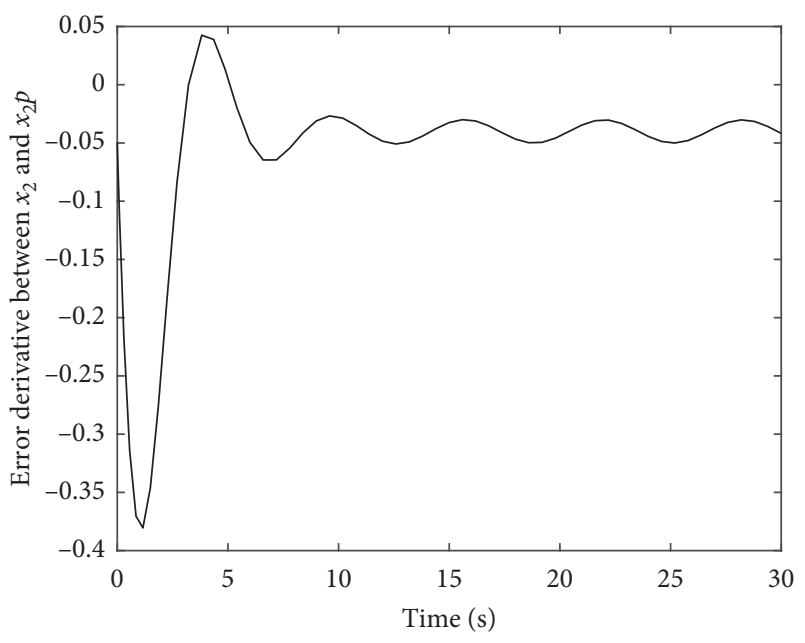

(b)

Figure 2: State observation error.

Figure 2(b), the error gradually stabilizes and is near 0 . It can be seen that the system has a good tracking effect.

In Figure 3(a), it can be seen that the position tracking error is relatively large in the first 4.3 seconds, and in the subsequent time, the position tracking has achieved good results. In Figure 3(b), for the speeding tracking, the margin of error is large in the first 2.6 seconds, which drops from 5.7 to 0.1 , and the error tends to 0 after 2.6 seconds, and the effect of speed tracking becomes better.

In Figure 4(a), for $x_{1}$ estimation error, the error range goes from 0.5 to -0.223 in the first 7.9 seconds. It goes from -0.223 to -0.011 , and the error is about -0.01 after
7.9 seconds. In Figure 4(b), for the estimation error of $x 2$, the error has a relatively large change in the first 7.52 seconds. After 7.52 seconds, the error gradually stabilizes.

As shown in Figure 5, the control input has a relatively large change in the first 2.3 seconds, and its amplitude reaches 78 . The control input tends to be stable gradually after 2.3 seconds.

The simulation results demonstrate that the degree of coincidence was gradually improved. In a word, the vehicle speed system has obtained stable control, and the position and speed have good tracking effects. 


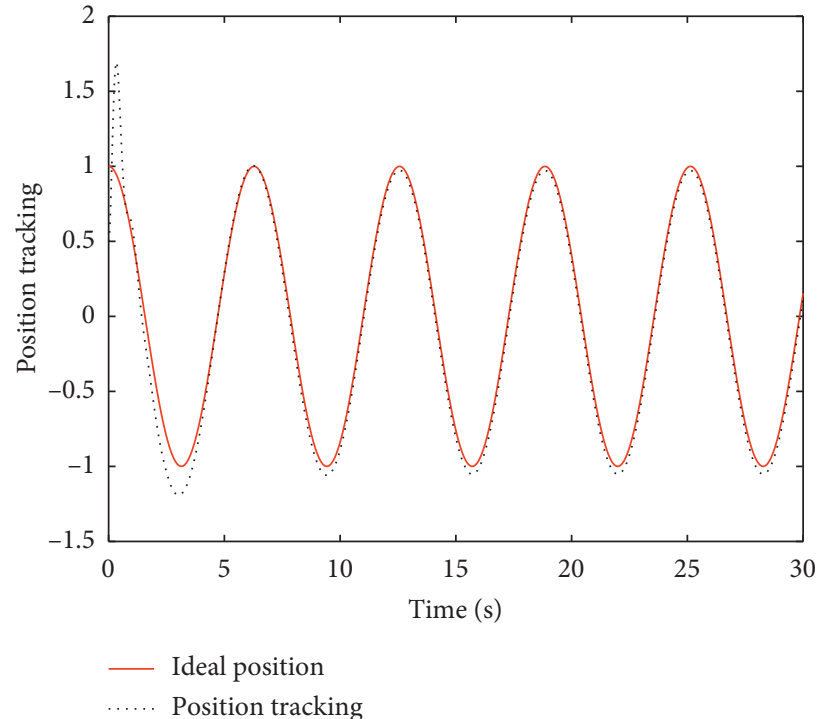

(a)

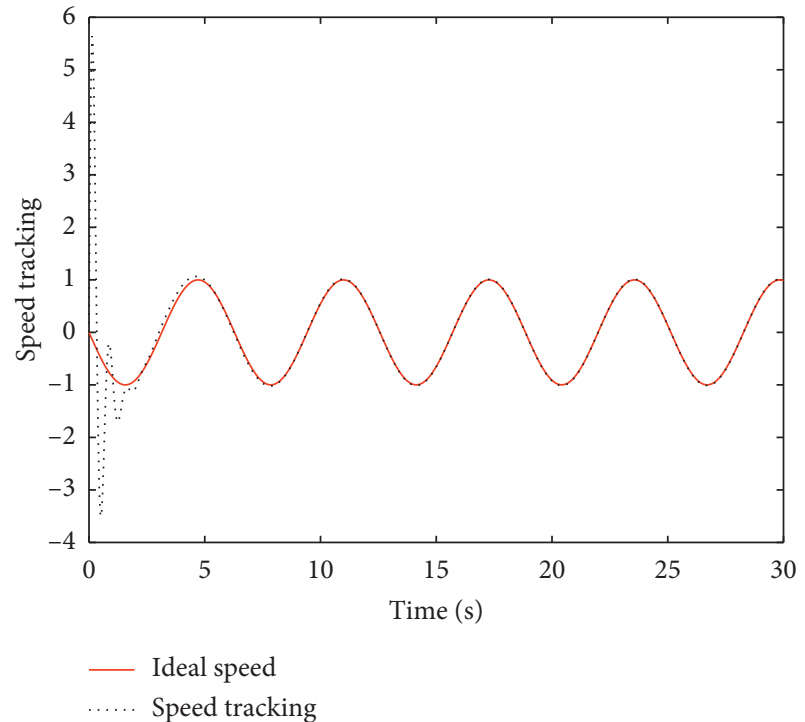

(b)

Figure 3: Position and speed tracking.

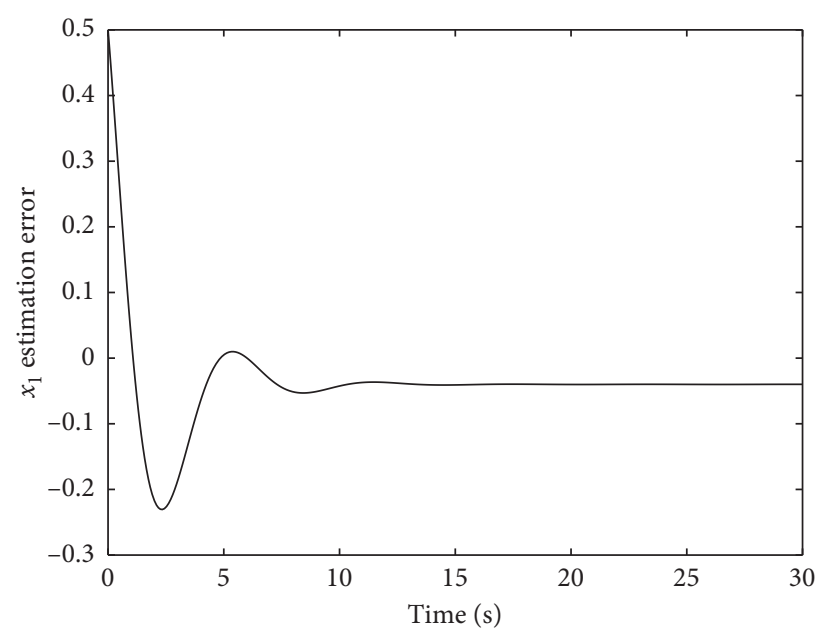

(a)

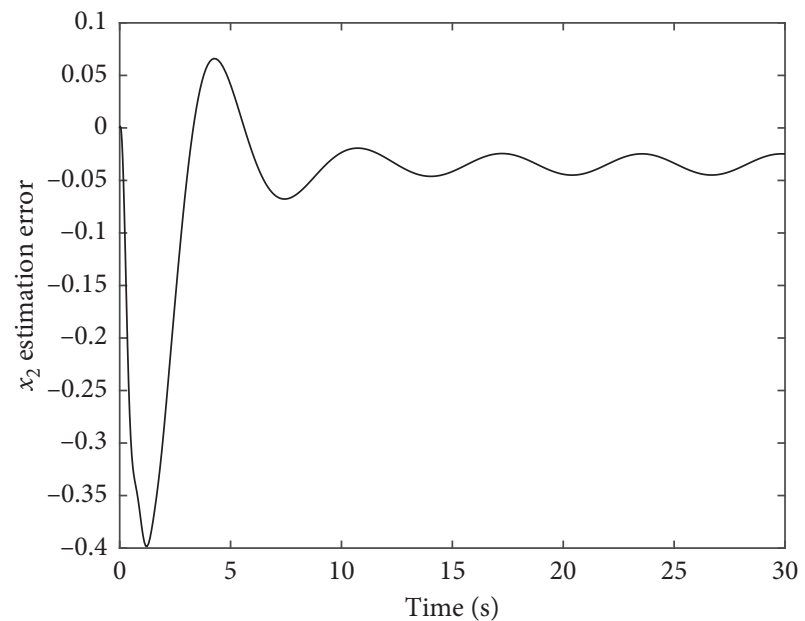

(b)

Figure 4: Estimation error of $x_{1}$ and $x_{2}$.

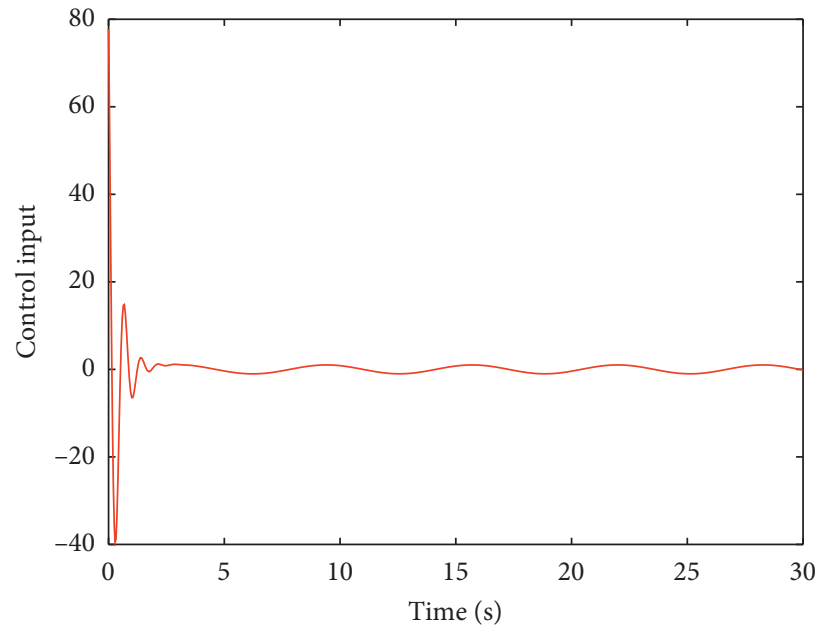

Figure 5: Control input. 


\section{Conclusion}

To conclude, the vehicle speed system in actual operation has strong nonlinearity. For low-speed motion, a simple controller can achieve better control effect, but for high-speed motion, a simple controller is difficult to obtain good control effect. Therefore, a more appropriate controller should be used. In such a case, a sliding mode control method based on LMIs is proposed to improve the stability and traceability of the vehicle speed system. In this process, the SSM is designed to improve the accuracy of the controller. Therefore, the accuracy of system tracking is ensured. In the meanwhile, the observer gain matrix algorithm is designed in the study of sliding mode control of the nonlinear system, and the obtained observer gain matrix ensures the stable operation of the system. According to simulation results, the proposed method shows a good control effect on the vehicle speed system.

\section{Data Availability}

No data were used to support this study.

\section{Conflicts of Interest}

The authors declare that there are no conflicts of interest regarding the publication of this paper.

\section{Acknowledgments}

This work was supported by the National Natural Science Foundation of China (Grant nos. 61971181 and 61602163) and the Natural Science Foundation of Hubei Province (Grant no. 2016CFC735).

\section{References}

[1] T. Han and W. X. Zheng, "Bipartite output consensus for heterogeneous multi-agent systems via output regulation approach," IEEE Transactions on Circuits and Systems II: Express Briefs, vol. 68, no. 1, pp. 281-285, 2021.

[2] T. Han, B. Xiao, X. S. Zhan, and H. Yan, "Bipartite containment of descriptor multi-agent systems via an observerbased approach," IET Control Theory \& Applications, vol. 14, no. 19, pp. 3047-3051, 2020.

[3] L. Hao, X. Zhan, J. Wu, T. Han, and H. Yan, "Bipartite finite time and fixed time output consensus of heterogeneous multiagent systems under state feedback control," IEEE Transactions on Circuits and Systems II: Express Briefs, vol. 99, p. 1, 2020.

[4] H.-L. Gao, X.-S. Zhan, Y.-R. Yuan, Z.-J. Pan, and G.-L. Yuan, "Mitigation of low frequency oscillations in power systems based on Mamdani fuzzy inference," Transactions of the Institute of Measurement and Control, vol. 41, no. 12, pp. 3477-3489, 2019.

[5] M. R. Tavana, M.-H. Khooban, and T. Niknam, "Adaptive PI controller to voltage regulation in power systems," ISA Transactions, vol. 66, pp. 325-334, 2016.

[6] H. J. Clayton, L. Ilias, and S. Dragos, "Conceptual design, flying, and handling qualities assessment of a blended wing body (BWB) aircraft by using an engineering flight simulator," Aerospace, vol. 7, no. 5, pp. 1-19, 2020.
[7] H. Gao, X. Li, and C. Wu, "Neural network supervision control strategy for inverted pendulum tracking control," Discrete Dynamics in Nature and Society, vol. 2021, pp. 1-14, 2021.

[8] F. Gouaisbaut, M. Dambrine, and J. P. Richard, "Robust control of delay systems: a sliding mode control design via LMI," Systems \& Control Letters, vol. 46, no. 4, pp. 219-230, 2002.

[9] D. Yang, G. Qiu, X. D. Zhao et al., “Terminal sliding mode control with adaptive feedback control in a class of chaotic systems," Mathematical Problems in Engineering, vol. 2014, Article ID 267024, 6 pages, 2014.

[10] S. Mobayen, D. Baleanu, and F. Tchier, "Second-order fast terminal sliding mode control design based on LMI for a class of non-linear uncertain systems and its application to chaotic systems," Journal of Vibration and Control, vol. 23, no. 13, pp. 2912-2925, 2016.

[11] H. Y. Su, R. Q. Lu, and X. Y. Zhang, "Second-order integral sliding mode control for uncertain systems with control input time delay based on singular perturbation approach," IEEE Transactions on Automatic Control, vol. 60, no. 11, pp. 3095-3100, 2015.

[12] S. Mobayen, "Design of LMI-based global sliding mode controller for uncertain nonlinear systems with application to Genesio's chaotic system," Complexity, vol. 21, no. 1, pp. 94-98, 2014.

[13] L. X. Zhang, L. G. Wu, C. H. Wang et al., "Sliding mode $H_{\infty}$ control for a class of uncertain nonlinear state-delayed systems," Systems Engineering and Electronic Technology, vol. 17, no. 3, pp. 576-585, 2006.

[14] X. Fan, Q. Zhang, and J. Ren, "Event-triggered sliding mode control for discrete-time singular system," IET Control Theory \& Applications, vol. 12, no. 17, pp. 2390-2398, 2018.

[15] S. Mobayen, "Design of LMI-based sliding mode controller with an exponential policy for a class of underactuated systems," Complexity, vol. 21, no. 5, pp. 117-124, 2014.

[16] X. Chen, H. Zhao, H. Sun, S. Zhen, and A. A. Mamun, "Optimal adaptive robust control based on cooperative game theory for a class of fuzzy underactuated mechanical systems," IEEE Transactions on Cybernetics, pp. 1-13, 2020.

[17] Q. Yao, "Adaptive fuzzy neural network control for a space manipulator in the presence of output constraints and input nonlinearities," Advances in Space Research, vol. 67, no. 6, pp. 1830-1843, 2021.

[18] J. Li, J. Ding, T. Chai, F. L. Lewis, and S. Jagannathan, "Adaptive interleaved reinforcement learning: robust stability of affine nonlinear systems with unknown uncertainty," IEEE Transactions on Neural Networks and Learning Systems, pp. 1-11, 2020.

[19] F. Tao, M. Li, and Z. Fu, “Operator-based robust stability for nonlinear systems with multiple uncertainties using coprime factorisation method," International Journal of Systems Science, vol. 52, no. 1, pp. 1-11, 2021.

[20] L. L. Li and D. Voskov, "A novel hybrid model for multiphase flow in complex multi-scale fractured systems," Journal of Petroleum Science and Engineering, vol. 203, no. 2, pp. 1-13, 2021.

[21] G. Zhao, H. Li, and T. Hou, "Input-output dynamical stability analysis for cyber-physical systems via logical networks," IET Control Theory \& Applications, vol. 14, no. 17, pp. 2566-2572, 2020.

[22] C. M. Agulhari and P. L. D. Peres, "Computing stabilising output-feedback gains for continuous-time linear timevarying systems through discrete-time periodic models," 
International Journal of Control, vol. 94, no. 3, pp. 687-697, 2021.

[23] G. Pin, M. Filippo, F. A. Pellegrino et al., "Approximate offline receding horizon control of constrained nonlinear discrete-time systems: smooth approximation of the control law," in Proceedings of the IEEE 2010 American Control Conference (ACC), pp. 6268-6273, Baltimore, MD, USA, June 2010.

[24] J. Yu, L. Zhao, H. Yu, and C. Lin, "Barrier Lyapunov functions-based command filtered output feedback control for full-state constrained nonlinear systems," Automatica, vol. 105, pp. 71-79, 2019.

[25] D. A. Muñoz and W. Marquardt, "Robust design of closedloop nonlinear systems with input and state constraints," IFAC Proceedings Volumes, vol. 45, no. 15, pp. 916-921, 2012.

[26] M. Gurtner, P. Zips, M. Atak, J. Ophey, and A. Kugi, "Improved EMD-based oscillation detection for mechatronic closed-loop systems," IFAC-PapersOnLine, vol. 52, no. 15, pp. 370-375, 2019.

[27] F. Beltran-Carbajal, R. Tapia-Olvera, A. Valderrabano-Gonzalez, H. Yanez-Badillo, J. C. Rosas-Caro, and J. C. Mayo-Maldonado, "Closed-loop online harmonic vibration estimation in DC electric motor systems," Applied Mathematical Modelling, vol. 94, pp. 460-481, 2021.

[28] N.-N. Jin, S. Liu, and H.-S. Zhang, "Linear optimal estimation for discrete-time and continuous-time systems with multiple measurement delays," International Journal of Control, Automation and Systems, vol. 19, no. 3, pp. 1194-1204, 2021.

[29] S. M. RakhtAla, M. Yasoubi, and H. HosseinNia, "Design of second order sliding mode and sliding mode algorithms: a practical insight to DC-DC buck converter," IEEE/CAA Journal of Automatica Sinica, vol. 4, no. 3, pp. 483-497, 2017.

[30] X. H. Yu, Z. G. Zeng, J. J. Wang et al., "Event-triggering load frequency control for multi-area power systems with communication delays," IEEE Transactions on Industrial Electronics, vol. 63, no. 2, pp. 1308-1317, 2015.

[31] H. F. Luo and I. M. Hashem, "A new robust LMI-based model predictive control for continuous-time uncertain nonlinear systems," Computing and Communications, vol. 61, no. 4, pp. 670-681, 2020.

[32] C.-H. Lien, "An efficient method to design robust observerbased control of uncertain linear systems," Applied Mathematics and Computation, vol. 158, no. 1, pp. 29-44, 2004.

[33] C.-H. Lien, "Robust observer-based control of systems with state perturbations via LMI approach," IEEE Transactions on Automatic Control, vol. 49, no. 8, pp. 1365-1370, 2004.

[34] F. Mesquine and H. Khallouk, "Robust stabilization in LMI regions for constrained input systems," IEEE Transactions on Circuits and Systems II: Express Briefs, vol. 66, no. 11, pp. 1840-1844, 2019.

[35] J. K. Liu and Z. J. Liu, Control System Design, Analysis and MATLAB Simulation Based on LMI, Tsinghua University Press, Beijing, China, 2020. 